\title{
Evaluating a Social Network Analytic Tool to Support Outbreak Management and Contact Tracing in an Outbreak of Pertussis
}

\author{
Esther Munene*, S. Mottice and J. Reid \\ Utah Department of Health, SIc, UT, USA
}

\section{Objective}

To determine the feasibility and value of a social network analysis tool to support pertussis outbreak management and contact tracing in the state of Utah.

\section{Introduction}

Pertussis (i.e., whooping cough) is on the rise in the US. To implement effective prevention and treatment strategies, it is critical to conduct timely contact tracing and evaluate people who may have come into contact with an infected person. We describe a collaborative effort between epidemiologists and public health informaticists at the Utah Department of Health (UDOH) to determine the feasibility and value of a network-analytic approach to pertussis outbreak management and contact tracing.

\section{Methods}

The partnership: In early 2012, epidemiologists from UDOH's Vaccine Preventable Disease Program and UDOH's public health informaticists formed a partnership to determine the feasibility and value of the Organizational Risk Analyzer (ORA) in pertussis outbreak management and contact tracing (1). Both entities have a longstanding partnership. A characteristic that has made the collaboration particularly strong and mutually beneficial is that both partners have expertise in disease surveillance and outbreak management. In addition, the informaticists have expertise in devising systems that help frontline healthcare providers.

The Organizational Risk Analyzer (ORA): ORA is a computational tool that extends network analysis by using a meta-matrix model. A meta-matrix is defined as a network of connecting entities. The tool uses one or more matrices in an organization's meta-matrix as input. From this input the tool calculates measures that describe the relationships and ties among the entities. ORA contains over 50 network and node level measures which are categorized by the type of risk they detect (1).

Procedures: Following approval from UDOH's Institutional Review Board, we analyzed records from 629 deidentified pertussis patients from the UT-NEDSS database from January 2011 to December 2011. The test data included demographics and epidemiological information. We used Excel to create .csv data files, uploaded the data into ORA, and displayed the data in meta-matrices consisting of nodes (cases/contacts) and edges (relationships). We used ORA's visualizer to check for data-entry errors before performing the network analysis.

Data Analysis: ORA's centrality measures (degree, closeness, betweenness, hub, and eigenvector) were used to identify geographic locations with high infection rates and the patients who were central to sustaining the outbreak. Next, we applied a concor algorithm to find groups in the meta-network that might be hard to spot visually. Visualizations were used to supplement the metrics

\section{Results}

The ORA analysis identified 5 individuals who were central to perpetuating the outbreak in that their centrality measures were higher than other patients in the network. The index patient (Fig 1) was traced back to Utah County and was linked to 6 direct contacts in the same county and several indirect ties in adjacent counties. The individual was highly connected to others within the network (hub centrality $=1.41$ and eigenvector centrality $=1.00$ ). Salt Lake County had the highest number of cases, followed by Utah County and Weber County. The concor analysis revealed hidden networks, including a cluster of patients grouped by age group and case status (Fig 2).

\section{Conclusions}

The ORA was found to be a valuable tool for supporting pertussis outbreak management and contact tracing. Although network analysis is relatively new to public health, it can increase public health's understanding of how patterns of social relationships can aid or inhibit the spread of communicable diseases and provide the information needed to target intervention efforts effectively.

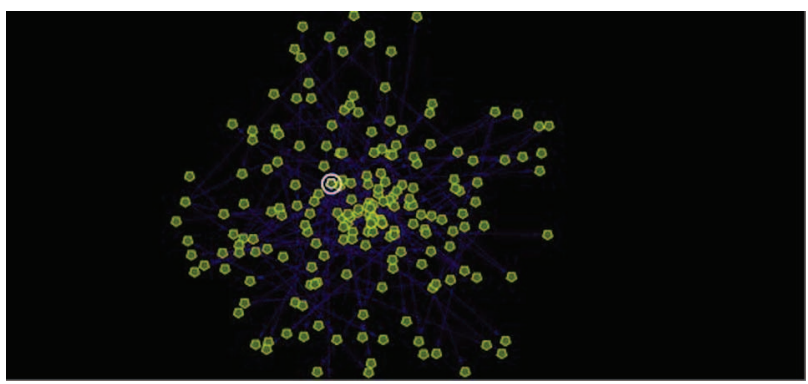

Fig. 1. The index case.

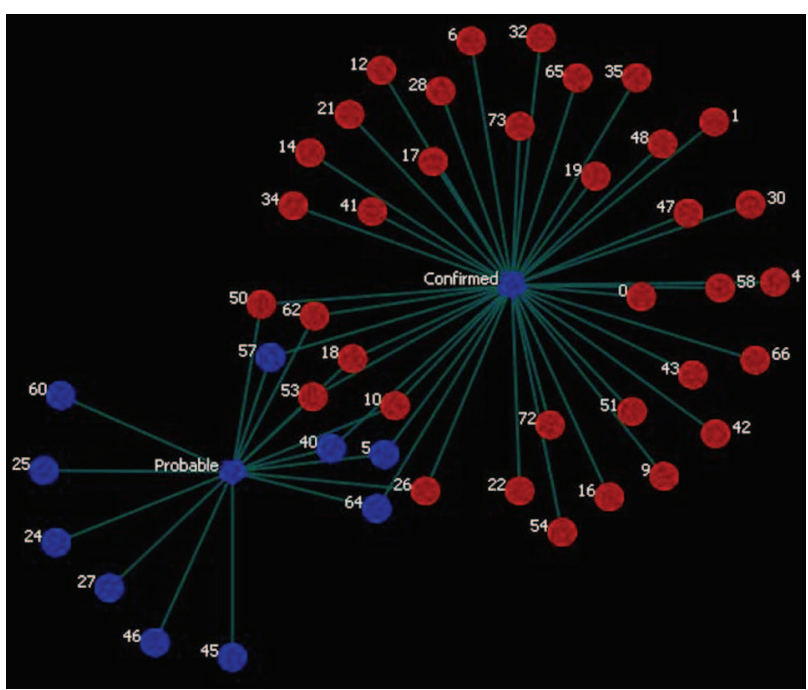

Fig. 2. Concor cluster of patients, by age group and case status. 
Keywords

Surveillance; Informatics; Social Network Analysis

\section{Acknowledgments}

This study was supported in part by the CSTE Applied Public Health Informatics Fellowship program and funded by the CDC.

\section{References}

1. Organizational Risk Analyzer: Center for Analysis of Social and Organizational Systems 2008.

\section{*Esther Munene}

E-mail: EMUNENE@utah.gov 